\title{
A Theory of Coordination: From Propositions to Hypotheses in Agile Software Development
}

\author{
Geetha Kanaparan \\ Xiamen University Malaysia \\ geetha.kanaparan@xmu.edu.my
}

\author{
Diane E. Strode \\ Whitireia New Zealand \\ diane.strode@whitireia.ac.nz
}

\begin{abstract}
Coordination is crucial in agile software development projects and a Theory of Coordination in co-located agile software development projects explains coordination in this context. This theory has propositions based on case study research. To improve the generalisability of theory built from case studies, researchers often transition to a theory testing phase involving a large-scale field study using the survey method. Prior to a large-scale field study, the propositions generated during theory building must be converted to testable hypotheses. There is little guidance explaining the complexity of this transition process and the challenges involved. Therefore, this paper explains the operationalisation process of transitioning from research propositions to research hypotheses and illustrates the process using the Theory of Coordination. The paper offers six practical guidelines, identifies seven challenges encountered, and potential solutions for each challenge. This paper contributes to agile software development and theory testing research offering seven recommendations for research practice.
\end{abstract}

\section{Introduction}

Agile software development is a philosophy and practices for organising the development of information systems. This paper is motivated by three issues in information systems research. Firstly, we identify a need to extend the generalisability of a significant Theory of Coordination in co-located agile software development projects used in information systems development and software engineering [1]. This theory, authored by [1] has had considerable impact. The theory has provided an analytical framework to study agile software development projects, is used to explain agile project management, and has contributed to tool development [2]-[5]. However, the theory is built from a small number of cases and has never been tested in a large- scale field study. For brevity, we refer to this theory as the Theory of Coordination in this paper.

Secondly, we identify a lack of guidelines on how to operationalise the research propositions of a theory such as the Theory of Coordination, into a set of hypotheses that can be empirically tested in a large-scale field study using a survey questionnaire. There is little guidance explaining the complexity of this process and the issues involved. This transition from theory building to theory testing is often treated without detail in current guidelines for mixed methods research, where issues such as deficiencies in the testable research model [6] and poorly defined constructs in scale development procedures [7] are commonly reported. Therefore, in this paper, we offer detailed practical guidance on this transition using the Theory of Coordination to illustrate the process. To operationalise the research propositions, this paper expands the framework for integrating case study research with survey methods proposed by [8] and extends the theory testing process proposed by [9].

Thirdly, during the operationalisation process, we identify challenges faced in the transition from theory building to theory testing research. We illustrate these challenges as they occur for the Theory of Coordination and offer solutions for each challenge. We also offer recommendations for research practice.

To guide the research, the research question for this study is, therefore:

What is the process to transition from research propositions to testable research hypotheses for the Theory of Coordination in agile software development projects?

This paper contributes to agile software development because we illustrate the complexity of generalising an existing theory, relevant to agile software development, to multiple agile contexts. The paper also contributes to the practice of theory building and theory testing because we provide detailed guidelines on how to transition from propositions generated during theory building to hypotheses testable in the theory testing phase of scientific research. 
This paper is organised as follows. First, we explain the current status of agile software development research and the Theory of Coordination. We then explain the issue of theory building from case studies which generate conceptual models and propositions and how this research fits with theory testing research using large-scale surveys. The next section sets out guidelines for the transition from propositions to testable hypotheses using the Theory of Coordination to illustrate the process. Following this, we set out the challenges encountered during this transition and propose solutions. We discuss the contributions of the paper and make seven recommendations for research practice.

\section{Background}

\subsection{Coordination theory in agile software development}

Agile software development has created a paradigm shift in the way software-intensive systems are developed [10]. In the early 2000s, agile methods such as Extreme Programming and Scrum were novel; in the 2020s, agile methods are not only the most common approach for small co-located projects they are increasingly the preferred approach in large-scale, and globally distributed systems development projects [11][13].

Coordination is crucial to the success of all forms of software development including agile software development [14], [15]. A theoretical model of coordination in co-located agile software development projects was developed based on empirical evidence from three case studies [1]. The cases used the agile methods Scrum (2 cases) and Scrum with practices from Extreme Programming (1 case).

To develop the theoretical model in [1], the authors followed guidelines for building theory from positivist case study research [16]-[20]. The original underpinning of this theory came from an interdisciplinary study of coordination proposed by [21] and elaborated by [21], [22]. This interdisciplinary study of coordination is based on the premise that in any coordination processes, dependencies occur that can be managed with coordination mechanisms [22], [23]. The Theory of Coordination proposes that agile software development projects might embody effective coordination, and after analysing the coordination mechanisms in three cases of agile software development [1] proposed that the coordination mechanisms present in agile software development projects form a coordination strategy.
According to the Theory of Coordination, a coordination strategy is a group of coordination mechanisms purposefully selected by the co-located agile project team to manage the dependencies in their project. Such dependencies are described in a taxonomy by [24]. To address these dependencies, agile software development methodologies (e.g. Scrum) provide a variety of coordination mechanisms such as task boards [25], specialised meetings [26], and colocation of teams [27]. Other coordination mechanisms that are not related to any particular methodology can also be used (e.g. online chat tools, automated regression tools). Together all of these coordination mechanisms form a project's coordination strategy. The Theory of Coordination identifies three coordination strategy components, that is, coordination mechanisms for synchronisation, for structure, and for boundary spanning. The full conceptual model is shown in Figure 1.

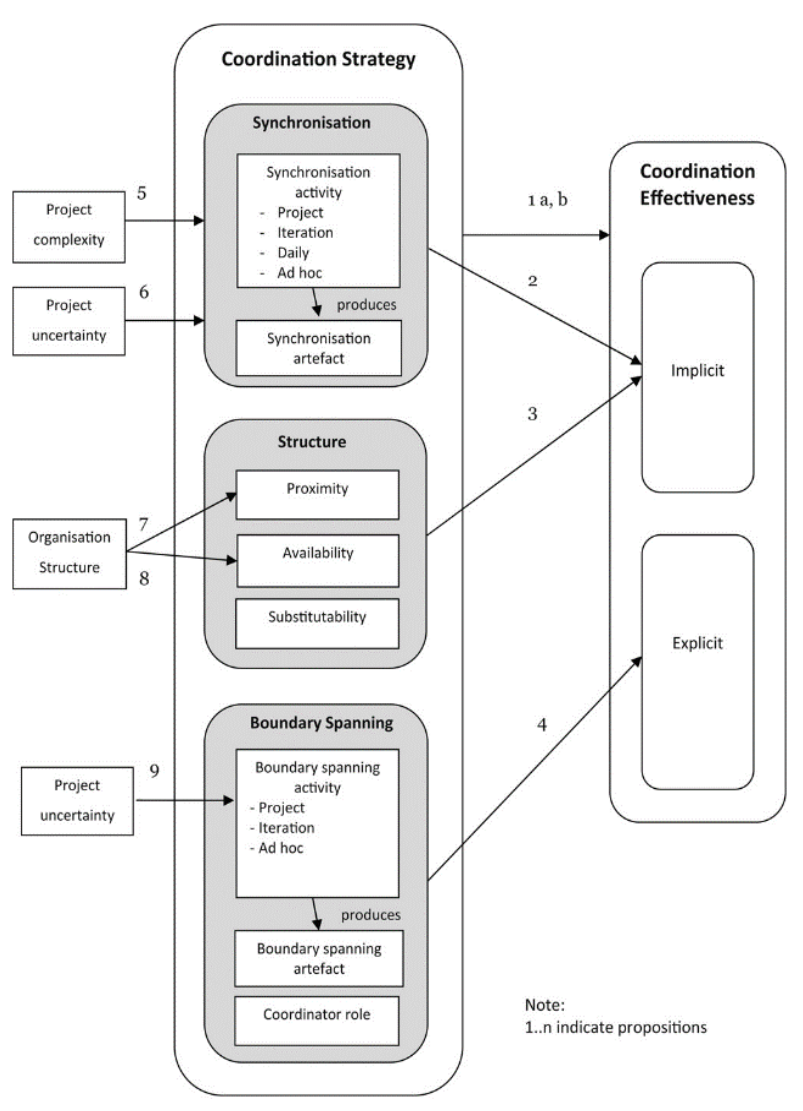

Figure 1. Theory of Coordination [1]

The purpose of synchronisation is to share knowledge and gain feedback in a project team. Synchronisation is "achieved with synchronisation activities and synchronisation artefacts produced and used during those activities" [1, p. 1230]. Synchronisation activities bring all project team 
members together at the same time and place for some pre-arranged purpose. A typical synchronisation activity is a planning meeting, retrospective, or daily stand up meeting. These activities occur at different frequencies: once per project, once per iteration, daily, and ad hoc (i.e. as and when necessary). These frequencies are due to the use of Scrum and sprints in the cases. Synchronisation artefacts are things produced and used during synchronisation meetings and include items such as designs, stories, and product and sprint backlogs.

Structure coordination mechanisms are concerned with the arrangement of the project team (i.e. intra-team structure). Three coordination mechanisms contribute to structural coordination: proximity (how close in space the team members are, which could be in a single room or more distributed), availability (how readily available team members are when they are needed, which is a function of workload, and full-time and part-time work arrangements) and substitutability (how readily team members can replace one another when needed, which is a function of their skill sets, which could be shared skill sets or highly specialised skill sets).

Boundary spanning is similar to synchronisation but involves interactions between the project team members and stakeholders or other teams involved in the project. Boundary spanning coordination mechanisms include both activities (e.g., meetings with stakeholders to discuss requirements or designs) and boundary spanning artefacts (e.g., sharing of projectrelated documents). When using a method such as Scrum with sprints, boundary spanning activities can occur once per project, once per iteration, daily, or ad hoc.

The outcome of an appropriate coordination strategy is an effectively coordinated project. In [1]'s theoretical model, the coordination effectiveness concept is defined as " $a$ state of coordination wherein the entire agile software development team has a comprehensive understanding of the project goal, the project priorities, what is going on and when, what they as individuals need to do and when, who is doing what, and how each individual's work fits in with other team members work. In addition, every object (thing or resource) needed to meet a project goal is in the correct place or location at the correct time and in a state of readiness for use from the perspective of each individual involved in the project" [28, p. 10]. Coordination effectiveness has two dimensions: implicit coordination and explicit coordination. Explicit coordination encompasses the physical objects (people or artefacts) involved in a project. When a project is coordinated effectively, required objects are in the correct place, at the correct time and in a state of readiness for use from the perspective of each individual involved in the project. Implicit coordination is concerned with coordination that occurs within workgroups without explicit speech or message passing and has the components: 'Know why', 'Know what is going on and when' 'Know what to do and when', 'Know who is doing what' and 'Know who knows what'.

The coordination theory for agile software development projects has been used in practical and theoretical ways in the fields of software engineering, information systems development, and IT project management. For example, ideas from [1]'s Theory of Coordination are used in a textbook for IT professionals on IT digitalisation where the authors replace project management and process management with coordination strategy and coordination effectiveness concepts [2]. The idea of mapping dependencies and coordination mechanisms has been used to identify dependencies and coordination mechanisms occurring in large-scale DevOps teams [3]. [4] use the definition of implicit coordination provided in the Theory of Coordination to inform the design of a communication support tool for agile projects. [5] studied agile software development coordination artefacts and used ideas from the Theory of Coordination as the basis for their study. Given this significant impact on industry practice and theory, the Theory of Coordination should be tested to provide evidence of its generalisability to agile contexts more broadly.

\subsection{Theory building to theory testing}

The domain of information systems has an established tradition of positivist research. This positivist research is primarily quantitative [29], but a small body of positivist research is qualitative [9], [16], [19], [20]. These two forms of positivist research differ in their goals. The goal of quantitative positivist research is to generalise research findings to populations of interest, and data analysis is primarily deductive involving the testing of theoretical propositions developed a priori. Qualitative positivist research has the goal of generalising research findings to theoretical concepts of interest and data analysis is primarily inductive and builds theory directly from empirical evidence. This distinction is not absolute; many studies combine quantitative and qualitative data, and deductive and inductive analysis [30], [31].

[17] explain how positivist qualitative research and positivist quantitative research fit together in building and testing theory. The positivist qualitative research methodology is appropriate to build theory by defining concepts or constructs (constructs are more precisely defined than concepts [32]) and propositions linking those concepts. Such theory can then be tested following a positivist quantitative research methodology. 
The Theory of Coordination is based on a positivist qualitative study. To generalize the findings from this theory, a positivist quantitative method using a largescale survey would be a commonly accepted technique to examine the strength of the relationship between Coordination Strategy and Coordination Effectiveness. This overarching research design would fit the corroboration/confirmation mixed methods approach proposed by [31]. This paper focuses on the transition phase from qualitative to quantitative research.

Despite compelling evidence that qualitative and quantitative research fit together in building and testing theory, there is a lack of advice and guidelines in the information systems (IS) literature on the transition process, although this process is common in the IS discipline and in other social science disciplines.

\section{Method}

Our starting point for transitioning the research propositions from the Theory of Coordination to testable research hypotheses was to apply and expand the procedure proposed by [8] for integrating case study with survey methods. [8]'s procedure is a series of stages for moving from conceptual models built from case studies, the operationalisation of variables and instrument design, through to the testing of hypotheses in a survey and interpretation of findings. We focus on stage 7 in [8]'s procedure; the operationalisation of variables, and expand on that stage with our own guidelines for this stage in the process.

While [8]'s paper focuses on the stages to integrate case study and survey methods, a related paper by [9] proposed a detailed 6-step extensive theory testing process using case study research which we were able to adapt and extend in our research. Although their proposed process used case studies to test the theory, we found the process suitable for our research which uses a quantitative method to test the theory. [9]'s theory testing process consists of the following steps:

1. Establish Theory

2. Design Case Study Research

3. Prepare for Data Collection and Analysis

4. Collect Empirical Data

5. Analyze Empirical Data

6. Extend the Theory

Of relevance to our research is the first step. Step 1 of the theory testing process, Establish Theory, is discussed in the following section.

\section{The transition from theory building to theory testing}

Step 1 of [9]'s theory testing process suggests that theory is established by a) establishing testable propositions, b) identifying causal mechanisms that affect results, and c) operationalising propositions to testable hypotheses with concrete indicators. Since the Theory of Coordination identified nine propositions (see Guideline 5), our paper focuses on identifying the causal mechanisms in the model (see Figure 1) and operationalises the propositions by applying a process for transitioning from the research propositions proposed during theory building to a set of testable hypotheses for theory testing. We propose guidelines based on common activities that are described extensively in the literature on quantitative research methods [7], [29], [33], [34]. However, those descriptions lack detail on a methodical approach to transition from theory building to theory testing. Our paper provides such detail. The guidelines are presented in the sequence that we took to transition from theory building to theory testing.

Guideline 1: Examine theoretical model for dependent and independent variables

A fundamental task in developing a testable theoretical model is to determine the independent and dependent variables. By identifying the independent and dependent variables, the research problem is then presented in a form that enables the presentation and evaluation of a cause and effect relationship. Our proposed quantitative study aims to determine the extent to which agile coordination contributes to the coordination effectiveness of software projects. Since the conceptual framework proposed for the Theory of Coordination posits that Coordination Strategy determines Coordination Effectiveness, Coordination Strategy was identified as the independent variable while Coordination Effectiveness was identified as the dependent variable.

\section{Guideline 2: Establish evidence of multi-dimensional variables in the theoretical framework}

Identifying the attributes or dimensions of a multidimensional variable is required for the measurement and operationalisation of the variable in a testable research model. Therefore, the theoretical framework was examined for possible multi-dimensional variables. A variable is multi-dimensional if it has "... a number of interrelated attributes or dimensions and exists in multidimensional domains" [35, p. 741] and may be “... distinguished between its levels of abstraction" [36, p. 370]. 
The Theory of Coordination shown in Figure 1 suggests that Coordination Strategy and Coordination Effectiveness are multi-dimensional variables with two levels of abstraction. The first level of abstraction for the multi-dimensional variable Coordination Strategy is Synchronisation artefacts, Synchronisation activity, Proximity, Availability, Substitutability, Boundary spanning activity, Boundary spanning artefact and Coordinator role. These variables may also be referred to as first-order variables. We excluded the Coordinator role from the testable model and we explain the reasons for the exclusion in section 5 on challenges encountered during the transition.

The second level of abstraction for the multidimensional variable Coordination Strategy is Synchronisation, Boundary Spanning and Structure which may also be referred to as second-order variables.

Similarly, the dependent variable, Coordination Effectiveness was conceptualised as a multidimensional variable. In Figure 1, Coordination Effectiveness appears to have been conceptualised to one level of abstraction. However, the text of the Theory of Coordination [1] explains that Coordination Effectiveness has two levels of abstraction. The first level of abstraction for the multi-dimensional variable Coordination Effectiveness consist of the following: Shared goal ('Know why'), Team situation awareness (comprising of 'Know what is going on and when', 'Know what to do and when', 'Know who is doing what'), Expertise location ('Know who knows what'), Right time, Right place and Right thing. The five implicit coordination effectiveness factors in the original model in [1], we re-labeled to Shared goal, Team situation awareness and Expertise location to provide more meaningful variable names.

The second level of abstraction for the multidimensional variable Coordination Effectiveness is Implicit and Explicit Effectiveness.

Guideline 3: Determine if the variables in the testable model are formative or reflective

With the dependent and independent variables and the multi-dimensional variables identified, the variables in the testable model were examined to determine if they should be measured formatively or reflectively. This decision has implications for the setup of the measurement model, the development of the measurement scale, and the types of analysis to perform during the data analysis stage. [3, p. 302] argue that the formative or reflective relationship between an indicator and a variable "... depends upon the researcher's theoretical expectations about how they should be related based on the conceptual definition of the construct.". [34] argue that the decision to measure reflectively or formatively may be considered from a theoretical and an empirical perspective. From a theoretical perspective, the factors to be considered are: a) the nature of the construct, b) the direction of the causality, and c) the characteristics of the indicators [34]. By applying the theoretical considerations proposed by [34], we concluded that all seven of the first-order variables for Coordination Strategy satisfied the considerations of a reflective measure. We reasoned that if there is a change in the variable, this will result in a change in the indicator which suggests that the direction of the causality flows from the first-order variables to the indicators. Further, the set of indicators that we developed to measure each of the first-order variables reflectively were interchangeable while preserving the content validity of the variable if any single indicator was included or excluded. These characteristics are indicative of a reflective measure.

The second-order variables for Coordination Strategy were also examined for their direction of causality. We found evidence in the Theory of Coordination to suggest that the direction of the causality is from the indicators (first-order variables) to the second-order variables which implies that a change in the indicators would cause a change in the variable; i.e. the opposite of reflective models. As an example, Structure (first-order variable) is made up of Proximity, Availability and Substitutability. A change in the Proximity variable would cause a change in the Structure variable. We concluded that the independent variable, Coordination Strategy, conforms to the Type II - Reflective First-Order, Formative Second-Order type of multi-dimensional models proposed by [33].

A similar decision was made for the dependent variable, Coordination Effectiveness, which is also a multi-dimensional variable in the Theory of Coordination. We concluded that Coordination Effectiveness conforms to Type II - Reflective FirstOrder, Formative Second-Order multi-dimensional model [33] based on strong indications presented in the Theory of Coordination that the direction of causality is from the first-order variables (Shared goal, Team situation awareness, Expertise location, Right time, Right place and Right thing) to the indicators, and the direction of causality is from the indicators to the second-order variables (Implicit and Explicit effectiveness).

\section{Guideline 4: Identify possible moderating and mediating relationships}

The next decision in the development of the testable research model was to determine if any moderating or mediating relationships exist between the dependent and independent variables. [37, p. 6] suggests that a moderator variable "... modifies the form or strength of the relation between an independent and dependent 
variable”, while a mediator variable changes the causal sequence with the presence of a (mediator) variable in between the independent and dependent variable. Proposition $1 \mathrm{a}$ and $1 \mathrm{~b}$ in the Theory of Coordination (see Guideline 5) states that the customer's involvement (whether within or external to the project) in the project influences the relationship between Coordination Strategy and Coordination Effectiveness. This assertion is consistent with the interpretation of a moderating relationship. Thus, Customer involvement is proposed as a moderating variable between Coordination Strategy and Coordination Effectiveness. The conceptualisation of Customer involvement is discussed in section 5, Challenge 1.

\section{Guideline 5: Develop the hypotheses}

The next decision in developing the testable model is to develop the hypotheses for relationships that we intend to examine. A hypothesis is an "...empirical formulation of propositions, stated as relationships between variables" [38, p. 2.3.1]. The research propositions proposed during the theory building phase were reviewed and operationalised into a set of testable hypotheses for theory testing.

The Theory of Coordination proposed nine propositions. The propositions were developed following a two-step process. First, general inductive coding of the case data [39], [40] using an initial coding frame to identify dependencies and their associated coordination mechanisms was carried out. This is necessary because a coordination mechanism is only legitimate if it addresses a dependency [22]. Second, the propositions were then developed from a cross-case analysis [1].

In developing the testable model, we focused on the first four propositions as these propositions are directly concerned with the relationship between Coordination Strategy and Coordination Effectiveness. Propositions 5 through to 9 focus on Project uncertainty, Project complexity and Organisation structure. How we dealt with these three factors is discussed in Guideline 6 and section 5, Challenge 5 .

We now discuss the decisions made when operationalising each of the propositions proposed during the theory building stage to the corresponding hypothesis proposed for theory testing. The following propositions are from [1].

\section{Proposition 1}

Proposition 1a. A coordination strategy that includes synchronisation and structure coordination mechanisms improves project coordination effectiveness when the customer is included in the project team. Synchronisation activities and associated artefacts are required at all frequencies - project, iteration, daily, and ad hoc.

Proposition 1b. A coordination strategy that includes synchronisation, structure, and boundary spanning coordination mechanisms improves project coordination effectiveness when the customer is an external party to the project. Synchronisation activities and associated artefacts are required at all frequencies - project, iteration, daily, and ad hoc. Boundary spanning activities and associated artefacts are required at all frequencies - project, iteration, and ad hoc.

Proposition 1a and $1 \mathrm{~b}$ describes a relationship between the second-order variables Coordination Strategy (ie. Synchronisation, Structure and Boundary spanning) and Coordination Effectiveness. However, the difference between the two propositions lies in the existence of the customer who may either be within or external to the project team. When a customer is external to a project team, the Boundary spanning coordination mechanism becomes an important factor in the relationship between Coordination Strategy and Coordination Effectiveness.

For theory testing, we rationalised that the customer's existence within or external to the project had to be re-conceptualised. The rationalisation is discussed further in section 5, Challenge 1. Additionally, in Guideline 4, we proposed Customer involvement as a moderating variable. We, therefore, propose the following hypotheses for Proposition 1a and 1b:

Hypothesis H1: Coordination Strategy has a positive effect on Coordination Effectiveness.

Hypothesis H2: Customer involvement influences the effect of Coordination Strategy on Coordination Effectiveness.

\section{Proposition 2}

Proposition 2. Synchronisation activities at all frequencies - project, iteration, daily, and ad hoc, along with their associated synchronization artefacts, increase implicit coordination effectiveness.

Synchronisation, a factor of Coordination Strategy, is proposed to increase Implicit Coordination, which is a factor of Coordination Effectiveness. Thus, we propose the following hypothesis for Proposition 2:

Hypothesis H3: Synchronisation has a positive effect on Implicit Coordination Effectiveness. 


\section{Proposition 3}

Proposition 3. Structural coordination mechanisms i.e. close proximity, high availability, and high substitutability, increase implicit coordination effectiveness.

Structure, a factor of Coordination Strategy, is proposed to increase Implicit Coordination, which is a factor of Coordination Effectiveness. We propose the following hypothesis for Proposition 3:

H4: Structure has a positive effect on Implicit Coordination Effectiveness

\section{Proposition 4}

Proposition 4. High levels of boundary spanning coordination mechanisms, i.e. boundary spanning activities at all frequencies - project, iteration, and ad hoc, their associated boundary spanning artefacts, and a coordinator role, increases explicit coordination effectiveness.

Boundary Spanning, a factor of Coordination Strategy, is proposed to increase Implicit Coordination, which is a factor of Coordination Effectiveness. We propose the following hypothesis for Proposition 4:

H5: Boundary Spanning has a positive effect on Explicit Coordination Effectiveness.

Guideline 6: Review literature and theoretical model for control variables and antecedents

The final guideline we propose is to review literature and the theoretical model for control variables and antecedents.

The Theory of Coordination does not propose any antecedents for coordination strategy or coordination effectiveness. However, the theory authors [1] suggest that Coordination Effectiveness is an antecedent to project success as a result of evidence presented in literature. We did not include project success as a consequence of coordination effectiveness in our testable research model and we discuss the reasons for this exclusion in section 5, Challenge 7.

The Theory of Coordination proposed that Project uncertainty, Project complexity and Organization structure influences the Coordination Strategy of a project. These three factors were considered as possible control variables that affect the relationship between Coordination Strategy and Coordination Effectiveness. Upon closer examination, Project complexity and Project uncertainty were not included as control variables. Reasons for the exclusion are discussed in section 5, Challenge 5 .
In contrast, Organisation structure was included as a control variable in the proposed research model since it influences the extent of Proximity and Availability of the agile project team members. A control variable is typically identified as an extraneous variable that is not important but may have an impact on the dependent variable [38]. In this regard, although Organisation structure has been conceptualised in the Theory of Coordination to influence two factors that form the independent variable, Coordination Strategy, we argue that the effect of Organisation structure on the Proximity and Availability of agile project teams would have an effect on the dependent variable, Coordination Effectiveness for theory testing purposes.

The six guidelines proposed in this section led to the development of the research model for theory testing shown in Figure 2.

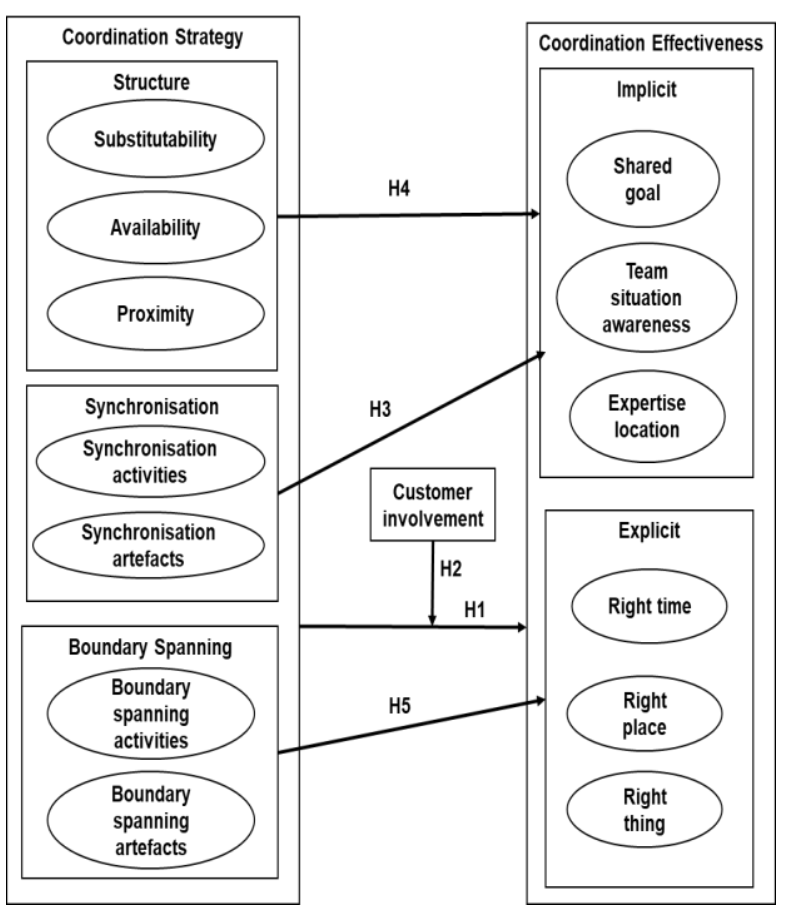

Figure 2. Proposed model for theory testing

\section{Challenges in the transition}

The transition from theory building to theory testing is not without its challenges. Parsimony had to be exercised to scope and model the in-depth and richly detailed findings from qualitative research into testable hypotheses for theory testing. In this section, we discuss the challenges we encountered and offer possible solutions. 


\section{Challenge 1: Customer Involvement} Solution 1: Re-conceptualise Relationships

The Theory of Coordination distinguishes a customer's involvement in the project based on whether they are within the project team or external to the project team. This is because a customer can be more or less involved in the work of the project team. A highly involved customer might be on site and work with the developer team daily (which is the preferred way to work on an agile team) [27], and this type of customer can be considered as 'internal' to the project team. In contrast, a much less involved customer might be quite distant physically from the team and not closely involved. For example, the customer contacts the team weekly or at unscheduled intervals, and this is considered as a customer who is external to the project team. For theory testing using a survey questionnaire, customer involvement may be described as a categorical variable. However, presenting customer involvement as a categorical variable may be a challenge as respondents of the survey questionnaire may not be able to clearly distinguish between an internal and an external customer. As a result, we re-conceptualised Customer involvement as a latent variable whereby their degree of involvement in the project will determine if the customer is on the internal or external end of the spectrum with regards to their involvement in the agile software project.

\section{Challenge 2: Excluding Coordinator role \\ Solution 2: Subsume Constructs}

Coordinator role was proposed as an indicator for Boundary spanning in the Theory of Coordination because of evidence from the literature indicating that people who took the role of coordinator act as conduits for the transfer of information between the agile project team and the customer group [41]. We excluded the Coordinator role as an indicator of Boundary spanning and instead subsumed the Coordinator role within the Boundary spanning activities. This is because, in a typical agile project, a specifically designated Coordinator role does not exist, although a Scrum master might take this role informally [25],[26].

\section{Challenge 3: Types of Agile Teams Solution 3: Extend Model Boundaries}

The Theory of Coordination was constructed based on co-located teams. Our proposed theory testing phase would allow for all types of agile software project teams. At the time the Theory of Coordination was proposed in 2012, agile approaches were largely practiced by co-located and small project teams. However, recent evidence from literature on agile projects suggest that agile methods are also used for large-scale and globally distributed projects [11]-[13].
In light of these findings, we decided to test the theory in any type of agile team. We propose that demographic data should be gathered on the scale and distribution of the agile team by asking questions on time zone differences, language differences within the teams, and the size of the agile team in a survey questionnaire.

\section{Challenge 4: Currency of Agile Projects Solution 4: Screen for Inclusion}

The cases that formed the basis of the Theory of Coordination were selected after satisfying the inclusion criterion that the project was current or recently completed. We considered the possibility of screening agile projects for a similar inclusion criterion for our theory testing phase. This could be achieved by advising the research participants to focus on a current project or a project that they recently completed when answering the survey questionnaire.

\section{Challenge 5: Excluding Project Complexity and Project Uncertainty Solution 5: Investigate Control Variables}

In Guideline 6, we deliberated on Project complexity and Project uncertainty as potential control variables. We decided to exclude these two variables as control variables in the testable research model for the following reasons. Agile software development research indicates that agile projects can accommodate uncertainty and complexity [12], [27], [42]-[44]. But the extent of Project uncertainty and complexity may change throughout a project. Since we intend to test the hypotheses with a cross-sectional survey questionnaire, this means that data will be gathered at one point in time, which could result in a biased interpretation for variables of this nature. In addition, the responses for these variables can be subjective in a questionnaire. Finally, the removal of complexity and uncertainty reduces the complexity of the testable model.

\section{Challenge 6: Activities and Artefacts}

\section{Solution 6: Rationalise Dependencies between Variables}

The Theory of Coordination proposed that Synchronisation activities produce Synchronisation artefacts. When considering these variables for theory testing, such a relationship could suggest that Synchronisation artefacts are dependent on Synchronisation activities. Nevertheless, we decided that both Synchronisation activities and Synchronisation artefacts are measured as two separate variables that measure Synchronisation in order to maintain parsimony on the proposed research model and to focus on the main purpose of investigating the relationship between Coordination Strategy and Coordination Effectiveness. The same observation was 
made on Boundary spanning, where Boundary spanning artefacts is dependent on Boundary spanning activities. A similar decision was made to measure Boundary spanning activities and Boundary spanning artefacts as separate variables that collectively measure Boundary spanning.

Challenge 7: The Role of Project Success

Solution 7: Scope Model Consequences and Antecedents

In the Theory of Coordination, the authors acknowledged that Coordination effectiveness is an antecedent to project success based on evidence from prior research. However, during the theory building phase, the relationship between Coordination effectiveness and project success and other antecedents were not examined in order to maintain focus on coordination [1]. As we transitioned into the theory testing phase, adequate scoping of the proposed testable research model was a factor to be considered. Project success was not modeled as a consequence of Coordination effectiveness as the aim of the theory testing phase was to examine the relationship between coordination strategy and coordination effectiveness. In addition, [1, p. 1226] argued that "... a project may be well coordinated yet be unsuccessful for reasons unrelated to coordination, such as misinterpretation of requirements, or budgetary and resource constraints."

\section{Discussion and conclusion}

This paper focused on the details of the transition from theory building to theory testing for the Theory of Coordination in agile software development projects (Figure 1). The main reason for the transition was to extend the generalisability of theory built from case study research to a large-scale field study. Existing research methods literature lacks detailed advice on the transition process, which motivated the need for our paper. We offer six detailed guidelines for transitioning from theory building to theory testing, with a focus on the activities and reasoning that occurs during the development of a testable research model. The guidelines are summarised as follows:

Guideline 1: Examine theoretical model for dependent and independent variables

Guideline 2: Establish evidence of multi-dimensional variables in the theoretical framework

Guideline 3: Determine if the variables in the testable model are formative or reflective

Guideline 4: Identify possible moderating and mediating relationships

Guideline 5: Develop the hypotheses
Guideline 6: Review literature and theoretical model for control variables and antecedents

Due to the richness and detail of theory built from qualitative research [17], we had to exercise parsimony in developing the testable research model (Figure 2) without compromising its content validity. We identified seven challenges during the transition process and offered solutions to overcome the challenges. Based on the solutions we developed, we offer seven recommendations for researchers who may experience similar challenges during the transition process:

Recommendation 1: Re-conceptualise Relationships Recommendation 2: Subsume Constructs

Recommendation 3: Extend Model Boundaries

Recommendation 4: Screen for Inclusion

Recommendation 5: Investigate Control Variables

Recommendation 6: Rationalise Dependencies between Variables

Recommendation 7: Scope Model Consequences and Antecedents

This paper makes two contributions to information systems. Firstly, this paper contributes to agile software development because we illustrate the complexity of generalising an existing theory, relevant to agile software development, to multiple agile contexts.

Secondly, this paper fills a gap in the literature, which includes deficiencies in the testable research model [6] and the need to improve construct definition for scale development [7], by providing guidelines on how to transition from propositions generated during theory building to testable hypotheses in the theory testing phase of scientific research. We also discuss potential challenges that researchers face in this phase of their research, provide solutions and offer recommendations.

This paper has limitations. The guidelines provided are particular to transitions from theory built from case studies to large-scale surveys and the illustration is focused on a single theory; the Theory of Coordination in agile software development projects. These guidelines have not been tested or applied to other methods of theory building (e.g. ethnography, phenomenology, action research, grounded theory) and theory testing (e.g. experimental research). Future research should address this limitation. Additionally, the challenges that occurred and the solutions that we proposed during the transition process are specific to our research. Although we make recommendations to overcome the challenges, further research is required to fully understand the transferability of our recommendations. 


\section{Acknowledgement}

This research was supported by Xiamen University Malaysia [XMUMRF/2020-C5/IECE/0015].

\section{References}

[1] D. E. Strode, S. L. Huff, B. Hope, and S. Link, "Coordination in Co-Located Agile Software Development Projects," J. Syst. Softw., vol. 85, no. 6, pp. 1222-1238, Jun. 2012, doi: 10.1016/j.jss.2012.02.017.

[2] C. T. Betz, Managing digital: Concepts and Practices. The Open Group Press, 2018.

[3] V. Stray, N. B. Moe, and A. Aasheim, "Dependency Management in Large-Scale Agile: A Case Study of DevOps Teams," in HICSS, 2019.

[4] S. Mastrogiacomo, S. Missonier, and R. Bonazzi, "Talk Before It's Too Late: Reconsidering the Role of Conversation in Information Systems Project Management," J. Manag. Inf. Syst., vol. 31, no. 1, pp. 47-78, Jul. 2014, doi: 10.2753/MIS0742-1222310103.

[5] A. Zaitsev, U. Gal, and B. Tan, "Coordination artifacts in Agile Software Development," Inf. Organ., vol. 30, Mar. 2020, doi: 10.1016/j.infoandorg.2020.100288.

[6] S. Gregor and G. Klein, "Eight Obstacles to Overcome in the Theory Testing Genre," J. Assoc. Inf. Syst., vol. 15, pp. I-XIX, Nov. 2014, doi: 10.17705/1jais.00382.

[7] S. B. MacKenzie, P. M. Podsakoff, and N. P. Podsakoff, "Construct Measurement and Validation Procedures in MIS and Behavioral Research: Integrating New and Existing Techniques," MIS Q., vol. 35, no. 2, pp. 293334, 2011.

[8] G. G. Gable, "Integrating case study and survey research methods: an example in information systems," Eur. J. Inf. Syst., vol. 3, no. 2, pp. 112-126, 1994, doi: 10.1057/ejis.1994.12.

[9] I. Chukwudi, M. Zhang, and G. Gable, "Extensive Theory Testing Using Case Study," in Proceedings of the 40th International Conference on Information Systems (ICIS 2019), United States of America: Association for Information Systems (AIS), 2019, pp. $1-17$.

[10] S. Denning, "The irresistible rise of agile: A paradigm shift in management," Forbes, 2019.

https://www.forbes.com/sites/stevedenning/2019/02/20/t he-irresistible-rise-of-agile-a-paradigm-shift-inmanagement/.

[11] VersionOne, "The 13th Annual state of agile report," 2019. https://www.stateofagile.com/\#ufh-i-52125190913th-annual-state-of-agile-report/473508.

[12] K. Conboy and N. Carroll, "Implementing Large-Scale Agile Frameworks: Challenges and Recommendations," IEEE Softw., Mar. 2019, doi: 10.1109/MS.2018.2884865.

[13] K. Dikert, M. Paasivaara, and C. Lassenius, "Challenges and success factors for large-scale agile transformations: A systematic literature review," J. Syst. Softw., vol. 119, pp. 87-108, 2016, doi: https://doi.org/10.1016/j.jss.2016.06.013.
[14] P. Dietrich, J. Kujala, and K. Artto, "Inter-Team Coordination Patterns and Outcomes in Multi-Team Projects," Proj. Manag. J., vol. 44, no. 6, pp. 6-19, Dec. 2013, doi: 10.1002/pmj.21377.

[15] R. E. Kraut and L. A. Streeter, "Coordination in software development," Commun. ACM, vol. 38, no. 3, pp. 69-82, 1995.

[16] K. M. Eisenhardt, "Building Theories from Case Study Research," Acad. Manag. Rev., vol. 14, no. 4, pp. 532550, 1989, doi: 10.2307/258557.

[17] K. M. Eisenhardt and M. E. Graebner, "Theory building from cases: Opportunities and challenges.," Acad. Manag. J., vol. 50, no. 1, pp. 25-32, 2007, doi: 10.5465/AMJ.2007.24160888.

[18] P. Darke, G. Shanks, and M. Broadbent, "Successfully completing case study research: combining rigour, relevance and pragmatism," Inf. Syst. J., vol. 8, no. 4, pp. 273-289, Oct. 1998, doi: 10.1046/j.13652575.1998.00040.x.

[19] L. Dubé and G. Paré, "Rigor in Information Systems Positivist Case Research: Current Practices, Trends, and Recommendations," MIS Q., vol. 27, no. 4, pp. $597-$ 636, Jul. 2003, doi: 10.2307/30036550.

[20] G. Paré, "Investigating Information Systems with Positivist Case Research," Commun. Assoc. Inf. Syst., vol. 13, p. 18, 2004.

[21] T. Malone, "What is coordination theory?," Massachusetts Inst. Technol. (MIT), Sloan Sch. Manag. Work. Pap., Jan. 1988.

[22] T. W. Malone and K. Crowston, "The Interdisciplinary Study of Coordination," ACM Comput. Surv., vol. 26, no. 1, pp. 87-119, Mar. 1994, doi: $10.1145 / 174666.174668$

[23] K. Crowston, J. Rubleske, and J. Howison, "Coordination Theory: A Ten-Year Retrospective," Former Dep. Centers, Institutes Proj., Oct. 2012.

[24] D. E. Strode, "A dependency taxonomy for agile software development projects," Inf. Syst. Front., vol. 18, no. 1, pp. 23-46, 2016, doi: 10.1007/s10796-0159574-1.

[25] “Agile practice guide," Project Management Institute, 2017. .

[26] K. Schwaber and J. Sutherland, "The Scrum guide," 2017. https://www.scrumguides.org/.

[27] K. Beck, "Embracing change with extreme programming," Computer (Long. Beach. Calif)., vol. 32, no. 10, pp. 70-77, 1999, doi: 10.1109/2.796139.

[28] D. Strode, B. Hope, S. Huff, and S. Link, Coordination Effectiveness In An Agile Software Development Context. 2011.

[29] D. Straub, M.-C. Boudreau, D. Gefen, A. 24. Communications of the Association for Information Systems: Vol. 13, D. 10.17705/1CAIS.01324, and A. at: Https://aisel.aisnet.org/cais/vol13/iss1/24, "Validation Guidelines for IS Positivist Research," Commun. Assoc. Inf. Syst., vol. 13, no. Article 24, 2004, doi: 10.17705/1CAIS.01324.

[30] A. S. Lee, "Integrating Positivist and Interpretive Approaches to Organizational Research," Organ. Sci., vol. 2, no. 4, pp. 342-365, Jul. 1991, [Online]. Available: http://www.jstor.org/stable/2635169. 
[31] V. Venkatesh, S. A. Brown, and H. Bala, "Bridging the qualitative-quantitative divide," Guidelines for conducting mixed methods research in information systems, vol. 37, no. 1. pp. 21-54, Mar. 2013, doi: 10.25300/MISQ/2013/37.1.02.

[32] R. Suddaby, "Challenges for Institutional Theory," $J$. Manag. Inq., vol. 19, no. 1, pp. 14-20, Feb. 2010, doi: $10.1177 / 1056492609347564$.

[33] C. Jarvis, S. MacKenzie, and P. Podsakoff, "A Critical Review of Construct Indicators and Measurement Model Specification in Marketing and Consumer Research," J. Consum. Res., vol. 30, pp. 199-218, Feb. 2003, doi: 10.1086/376806.

[34] T. Coltman, T. M. Devinney, D. F. Midgley, and S. Venaik, "Formative versus reflective measurement models: Two applications of formative measurement," J. Bus. Res., vol. 61, no. 12, pp. 1250-1262, Dec. 2008, doi: 10.1016/j.jbusres.2008.01.013.

[35] K. S. Law, C.-S. Wong, and W. H. Mobley, "Toward a Taxonomy of Multidimensional Constructs," Acad. Manag. Rev., vol. 23, no. 4, pp. 741-755, 1998, doi: 10.2307/259060.

[36] R. T. Wright, D. E. Campbell, J. B. Thatcher, and N. Roberts, "Operationalizing Multidimensional Constructs in Structural Equation Modeling: Recommendations for IS Research.," Commun. Assoc. Inf. Syst., vol. 30, 2012, [Online]. Available: https://doi.org/10.17705/1CAIS.03023.

[37] D. P. MacKinnon, "Integrating Mediators and Moderators in Research Design," Res. Soc. Work Pract., vol. 21, no. 6, pp. 675-681, Jul. 2011, doi: $10.1177 / 1049731511414148$.

[38] A. Bhattacherjee, Social Science Research - Principles, Methods, and Practices. University of South Florida: LibreTexts, 2020.

[39] M. B. Miles and A. M. Huberman, Qualitative data analysis: An expanded sourcebook, 2 nd ed. Thousand Oaks, CA, US: Sage Publications, Inc, 1994.

[40] D. R. Thomas, "A General Inductive Approach for Analyzing Qualitative Evaluation Data," Am. J. Eval., vol. 27, no. 2, pp. 237-246, Jun. 2006, doi: $10.1177 / 1098214005283748$.

[41] R. Hoda, J. Noble, and S. Marshall, "Self-Organizing Roles on Agile Software Development Teams," Softw. Eng. IEEE Trans., vol. 39, pp. 422-444, Mar. 2013, doi: 10.1109/TSE.2012.30.

[42] T. Little, F. Greene, T. Phillips, R. Pilger, and R. Poldervaart, "Adaptive agility," in Agile Development Conference, 2004, pp. 63-70, doi: 10.1109/ADEVC.2004.2.

[43] T. Dybå, T. Dingsøyr, and N. Moe, "Agile Project Management," in Software Project Management in a Changing World, R. G and C. Wohlin, Eds. Berlin, Heidelberg: Springer, 2014, pp. 277-300.

[44] D. Mishra and A. Mishra, "Complex software project development: agile methods adoption," J. Softw. Maint. Evol. Res. Pract., vol. 23, no. 8, pp. 549-564, Dec. 2011, doi: 10.1002/smr.528. 